\title{
Protective effect of androgens against inflammation induced cartilage degradation in male rodents
}

\author{
J A P Da Silva, J-P Larbre, T D Spector, L A Perry, D L Scott, D A Willoughby
}

Department of Experimental Pathology, St Bartholomew's Hospital Medical College,

London EC1M 6BQ, United Kingdom

J A P Da Silva

J-P Larbre

D A Willoughby

Department of

Reproductive

Physiology,

St Bartholomew's

Hospital Medical

College,

London EC1M 6BQ,

United Kingdom

L A Perry

Department of

Rheumatology,

St Bartholomew's

Hospital Medical

College,

London EC1M 6BQ,

United Kingdom

J A P Da Silva

T D Spector

D L Scott

Correspondence to:

Dr J A P Da Silva,

Department of

St Bartholomew's Hospital

Medical College,

Medical College,

London EC1M 6BO

United Kingdom.

Accepted for publication

9 December 1992

\begin{abstract}
Objectives-Rheumatoid arthritis (RA) is a disease which predominantly affects women. Interestingly, low serum androgen levels and clinical improvement with androgen replacement have been reported in male patients. The aetiopathogenic role of sex hormones in arthritis and their potential long term effects on joint destruction and disability remains unclear, however. This study was designed to investigate the potential influence of sex hormones on inflammation induced cartilage degradation in male rodents.
\end{abstract}

Methods-An in vivo model of cotton wrapped cartilage implants was used to assess the effects of androgen, oestradiol, and progesterone on inflammation induced cartilage degradation, and in vitro techniques were used to investigate the direct actions on cartilage metabolism and cytokine production in male animals.

Results-Orchidectomy resulted in accelerated cartilage damage which was reversed by replacement of physiological levels of androgens. Granulomatous tissue from castrated male rodents produced higher amounts of interleukin 1 . Sex hormones reduced spontaneous proteoglycan loss in vitro but did not interfere with the effects of interleukin 1 on cultured cartilage.

Conclusions-Androgens appear to protect cartilage from inflammation induced breakdown in male animals. These results support a pathogenic role for hypoandrogenism in rheumatoid arthritis and suggest that long term androgen replacement may help prevent joint damage and disability.

(Ann Rheum Dis 1993; 52: 285-291)

Rheumatoid arthritis (RA) is clearly affected by gender differences: the disease is not only two to three times more common in women, but also tends to be more aggressive and disabling in female patients despite an apparently balanced distribution of other risk factors. Furthermore, subclinical hypogonadism has been consistently reported in male patients ${ }^{12}$ and androgen replacement resulted in an improvement of immunological and clinical parameters of the disease. ${ }^{3}$ This is likely to be due to the immunosuppressive properties of androgens. ${ }^{4}$ Androgen receptors have been described in antigen presenting and processing cells in human synovia, suggesting a possible modulatory role at the core of local pathogenic mechanisms. ${ }^{5}$

Cartilage destruction is an early consequence of RA and plays a major part in the progression of disease and resulting disability. The effects of androgens on cartilage damage due to inflammatory processes have received little attention despite the demonstration of sex hormone receptors in articular cartilage from different species and experimental evidence that these hormones may stimulate proteoglycan and type II collagen synthesis by cultured human articular chondrocytes. ${ }^{6}$

The principal aim of the study was to investigate the influence of manipulation of androgens and other sex hormones on inflammation induced cartilage degradation in male animals.

\section{Methods}

IN VIVO CARTILAGE DEGRADATION

Castration and sham operation

Mature male BALB/c mice (Tuck \& Sons, United Kingdom) aged 9 to 10 weeks were used, age matched in each experiment, and kept under controlled light conditions (14 hours light, 10 hours dark). All surgical procedures were performed under intraperitoneal anaesthesia with $0.07 \mathrm{ml}$ of combined Hypnorm (Janssen) and Hypnovel (Roche) in water $(1: 1: 2, \mathrm{v} / \mathrm{v})$. Orchidectomy was carried out via a single abdominal incision after dissection and ligature of the spermatic cord. Sham operated animals had a similar operation but the gonads were replaced in the abdomen after exposure. Animals were left to recover for two weeks before further use.

Preparation of cartilage

Male Wistar rats $150-170 \mathrm{~g}$ (Tuck \& Sons) were killed and both femoral head cartilages (FHCs) were dissected free of bone and pooled in Hanks's balanced salt solution (HBSS) containing $100 \mathrm{IU} / \mathrm{ml}$ penicillin and $100 \mu \mathrm{g} / \mathrm{ml}$ streptomycin. The cartilages were thoroughly washed in HBSS, trimmed out of any adherent tissue in a laminar flow cabinet and left overnight in Dulbecco's modified Eagle's medium (DMEM), without phenol red supplemented with antibiotics as above, at 
$37^{\circ} \mathrm{C}$ in a humidified incubator $\left(5 \% \mathrm{CO}_{2} / 95 \%\right.$ air) before further use for implantation or culture.

\section{Cartilage implants}

Femoral head cartilages were aseptically prepared for implantation by wrapping in sterile surgical gauze (Softsorb, Vernon-Carus Ltd, United Kingdom) $(5 \pm 0 \cdot 15 \mathrm{mg}$ weight) moisturised with HBSS. Each mouse received a bilateral subcutaneous implant of either wrapped or non-wrapped FHC each side of the dorsal hump as described previously. ${ }^{7}$ A number of FHCs were kept frozen in DMEM for control purposes. At the end of the experiments the granuloma or non-wrapped cartilages were carefully dissected free from surrounding tissue. Femoral head cartilages were processed for glycosaminoglycan (GAG) analysis and the results are expressed as the percentage loss compared with frozen controls. The granulomas were weighed immediately and after drying for 48 hours at $56^{\circ} \mathrm{C}$ and the fluid volume and dry tissue mass were calculated.

\section{Assessment of levels of sex hormones in serum samples}

Levels of oestradiol were assessed using a commercially available radioimmunoassay (Diagnostic Products, Abingdon, United Kingdom). Serum progesterone was determined by radioimmunoassay as supplied by the North East Thames radioimmunoassay (NETRIA) unit. Testosterone was assessed by an inhouse radioimmunoassay as described previously. ${ }^{8}$ Interassay coefficients of variation for each steroid were less than $10 \%$ at three appropriate concentrations. The same testosterone assay was used to assess androgen levels after implantation of dihydrotestosterone capsules given that the assay shows $80 \%$ cross reactivity between the two steroids.

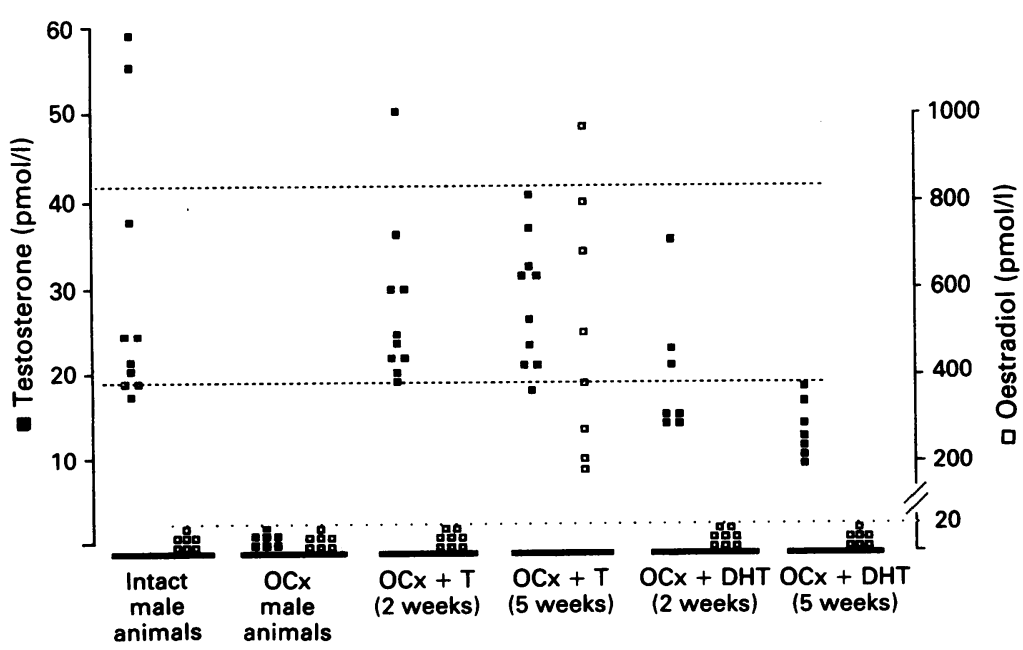

Figure 1 Androgen replacement with testosterone (T) and dihydrotestosterone (DHT) silastic capsules: effects on oestradiol levels. Serum testosterone (closed symbols) and oestradiol (open symbols) levels were measured in non-treated intact and orchidectomised (OCX) males as well as two and five weeks after subcutaneous implantation of $T$ and $D H T$ capsules. Broken lines represent the first and third quartile of $T$ levels in intact male animals. The dotted line represents the detection limit for oestradiol.

\section{Hormone treatment and replacement}

In all instances hormonal treatment, when used, was started immediately after the castration or sham operation. Control animals received the appropriate vehicle. In preliminary experiments oestradiol levels were shown to be below the detection limit of our assay $(20 \mathrm{pmol} / \mathrm{l})$ in all male animals and during most of the oestrus cycle in female animals. Treatment with this hormone was given by a biweekly subcutaneous injection of $0.5 \mu \mathrm{g} 17 \beta$ oestradiol in $0.05 \mathrm{ml}$ maize oil in the scruff of the neck. This was the lowest dose capable of inducing oestrus changes in vaginal smears of castrated female animals, the cyclical effects persisting effectively for at least five weeks (data not shown).

Progesterone was given in a subcutaneous silastic capsule implanted in the ventral surface of the animal. These were prepared using silastic tubing (inner diameter $1.6 \mathrm{~mm}$; outer diameter $2.4 \mathrm{~mm}$; Dow Corning, United Kingdom) sealed at both ends with silastic adhesive as to leave $15 \mathrm{~mm}$ free, packed with 15-17 mg of crystalline progesterone. This design was chosen from several tested and shown to replace physiological female levels (80-120 nmol/l) when implanted in castrated female animals, the concentration being stable between the second and fifth week after implantation.

Different designs of testosterone silastic capsules were tested. Figure 1 shows that testosterone capsules replacing physiological levels of this steroid resulted in unacceptably high levels of oestradiol, five weeks after implantation and were abandoned. Dihydrotestosterone subcutaneous silastic capsules (inner diameter $3.4 \mathrm{~mm}$; outer diameter 4.6 $\mathrm{mm} ; 20 \mathrm{~mm}$ free) packed with $70-75 \mathrm{mg}$ crystalline hormone resulted in stable androgen levels in the lower physiological range (allowing for the higher potency of dihydrotestosterone) with no detectable increase in oestradiol levels. Dihydrotestosterone was therefore used for androgen replacement.

\section{IN VITRO CULTURES}

Ex vivo interleukin 1 production by granulomatous tissue

Granulomatous tissue surrounding wrapped FHCs was carefully dissected out in a laminar flow hood 10 days after implantation and cultured in $2 \mathrm{ml}$ serum free DMEM for 48 hours. The supernatant was centrifuged, stored at $-70^{\circ} \mathrm{C}$, and interleukin 1 (IL-1) determined using a commercially available enzyme linked immunosorbent assay (ELISA) kit for mouse IL-1 $\alpha$ (Genzyme, United Kingdom), following the manufacturer's instructions. Interleukin 1 release was corrected for the granuloma dry weight.

\section{Cartilage culture}

Femoral head cartilages were cultured individually for 10 days in $2 \mathrm{ml}$ serum free and phenol red free DMEM supplemented with $100 \mathrm{IU} / \mathrm{ml}$ penicillin and $100 \mu \mathrm{g} / \mathrm{ml}$ streptomycin, and incubated at $37^{\circ} \mathrm{C}$ in a 
humidified incubator ( $5 \% \mathrm{CO}_{2} / 95 \%$ air), the medium being changed at day 5 . Sex hormones were added to the medium from stock solutions in dimethylsulphoxide (DMSO) to final concentrations chosen to cover a range from 10-fold below to 10-fold higher physiological levels in mature male Wistar rats as determined in preliminary experiments. The concentration of DMSO was kept at $0.01 \%$ in all groups.

When used, mouse recombinant interleukin $1 \alpha$ (Genzyme) prepared in DMEM with $0.5 \%$ bovine serum albumin (BSA) was added to the medium in the second five day period to a final concentration of $1 \mathrm{ng} / \mathrm{ml}$. A similar volume of $0.5 \%$ BSA was added to control wells. Cultured FHCs were pulsed with $37 \mathrm{kBq} / \mathrm{ml}$ carrier free $\mathrm{Na}_{2}{ }^{35} \mathrm{SO}_{4}$ (Amersham International, United Kingdom) for the last 24 hours of culture to evaluate proteoglycan synthesis. Supernatants were kept for the determination of GAGs.

\section{Assessment of cartilage and medium glycosaminoglycan}

Following implant or culture, FHCs were repeatedly washed and digested overnight at $56^{\circ} \mathrm{C}$ in $50 \mathrm{mM}$ phosphate buffer ( $\mathrm{pH} \mathrm{7.0)}$ containing $2 \mathrm{mM} \mathrm{N}$-acetyl cysteine, $1 \mathrm{mM}$ EDTA, and $6 \mathrm{U} / \mathrm{ml}$ papain. The GAG content of cartilage digests and culture supernatants was determined following the method of Farndale et $a l^{9}$ adapted to allow the use of a plate reader. Glycosaminoglycan loss from implanted FHCs was subsequently expressed as a percentage of the mean of frozen control cartilages. The percentage degradation of cultured FHCs was calculated according to the formula: (GAG medium/(GAG medium+GAG FHC) $) \times 100$.

\section{Assessment of proteoglycan synthesis in culture cartilage}

Pulsed FHCs were vigourously washed in cold $10 \mathrm{mM} \mathrm{MgSO}$ and digested in papain as described earlier. Aliquots of the digest were precipitated onto filter paper with $1 \%$ cetylpyridinium chloride and radioactivity in the precipitate was measured by liquid scintillation counting. Results are expressed in counts per minute (cpm) per milligram of cartilage weight after blot drying.

\section{Statistical methods}

Results are expressed as mean (SEM) values. Statistical significance was evaluated by the Mann-Whitney $U$ test for less than 30 samples and Student's $t$ test for larger numbers. Values of $\mathrm{p}<0.05$ (two tailed) were taken as significant.

\section{Results}

EFFECTS OF COTTON PELLET GRANULOMA ON CARTILAGE DEGRADATION

Groups of intact mice with a double implant of wrapped and non-wrapped FHC were killed at weekly intervals after implantation. Figure 2 shows that GAG loss increased progressively after the first week and the presence of cotton

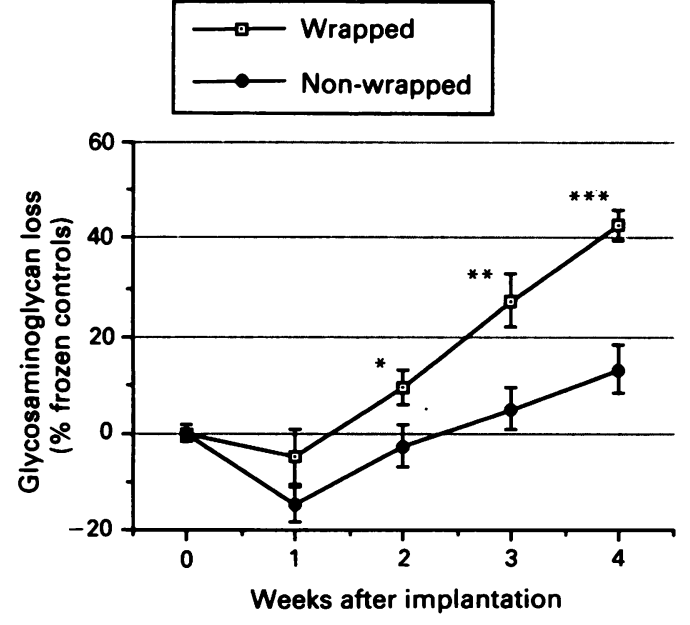

Figure 2 Effects of granulation tissue on the time course of glycosaminoglycan loss from implanted cartilage. Each mouse received a double subcutaneous implant of wrapped and non-wrapped femoral head cartilage. Groups of eight to 10 animals were killed at weekly intervals and the glycosaminoglycan content of cartilage was assessed and expressed by comparison with frozen cartilage. ${ }^{*} p=0.054$

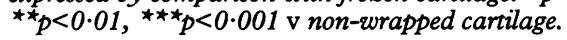

induced granulomatous tissue resulted in significant acceleration of cartilage destruction. Compared with frozen controls the GAG content of wrapped implanted cartilage showed a significant decrease from week 2 onwards, whereas this only barely achieved statistical significance at four weeks for non-wrapped cartilage. There was a moderate increase in the GAG content at week 1 which was significant for non-wrapped FHC $(p<0 \cdot 01)$. All subsequent experiments were stopped three weeks after implantation.

EFFECTS OF ORCHIDECTOMY ON CARTILAGE DEGRADATION IN THE PRESENCE AND ABSENCE OF GRANULOMA

In the absence of cotton induced granuloma, implanted cartilage lost less than $20 \%$ of the initial GAG content and this was not significantly changed by castration (fig 3 ). In the

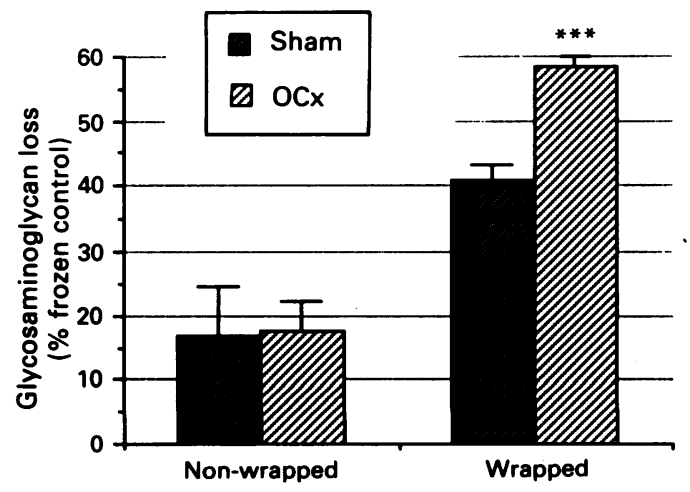

Figure 3 Effects of orchidectomy on glycosaminoglycan loss in the presence (wrapped; 11 animals) and absence (nonwrapped; 40-42 animals) of granulation tissue. Mice were castrated (OCX) or sham operated (Sham) two weeks before castrated (OCX) or sham operated (Sham) two weeks before
receiving a double implant of wrapped and non-wrapped femoral head cartilage. The glycosaminoglycan content of cartilage was assessed three weeks after implantation and compared with frozen controls. Pooled results from five different experiments. ${ }^{\star \star *} p<0.001 \mathrm{v}$ sham operated animals. 


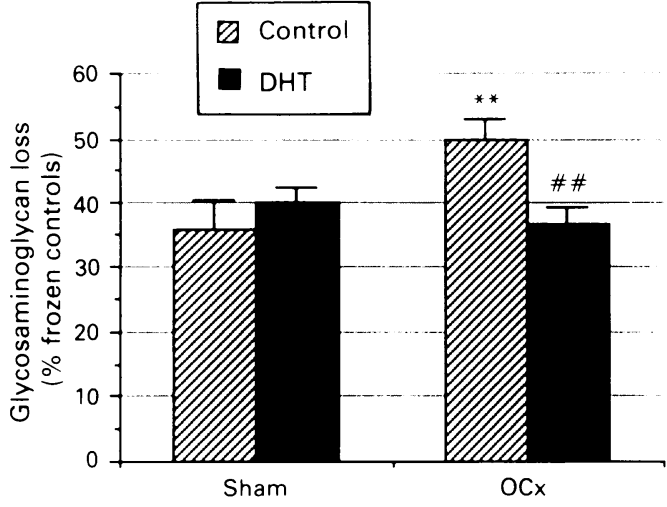

Figure 4 Effects of dihydrotestosterone (DHT) treatment on inflammation induced glycosaminoglycan loss. Animals had either an orchidectomy (OCX) or a sham operation (Sham) and immediately received a subcutaneous silastic capsule either empty (controls) or packed with DHT. Two weeks later each animal received an implant of cotton wrapped cartilage. Experiments were stopped three weeks after implantation, the glycosaminoglycan content of femoral head cartilages was assessed and compared with frozen cartilages. There were eight to 10 animals in each group. ${ }^{\star \star} p<0 \cdot 01 \mathrm{v}$ control sham. $\# \# p<0.01 \mathrm{v}$ control $O C X$

presence of granuloma, however, orchidectomy resulted in significantly accelerated cartilage degradation compared with sham operated animals $(59(2 \cdot 2) v 42(2 \cdot 2) \% ; \mathrm{p}<0.001)$. Bilateral cotton wrapped cartilage implants were used in subsequent experiments.

EFFECTS OF HORMONE TREATMENT AND REPLACEMENT ON CARTII AGE DEGRADATION Figure 4 shows the effect on GAG loss of dihydrotestosterone administration to sham operated and castrated male animals implanted with wrapped cartilages. Hormone replacement in castrated male animals resulted in a significant reduction of the rate of GAG loss from implanted cartilages $(36 \cdot 7 \quad(2 \cdot 7) \%)$ compared with castrated controls $(49 \cdot 8$ $(3.4 \%) ; \mathrm{p}<0.01)$ with complete reversal of the deleterious effects of castration. Treatment of sham operated animals did not confer further protection.

Figures 5 and 6 show the results of treatment of sham operated male animals and male

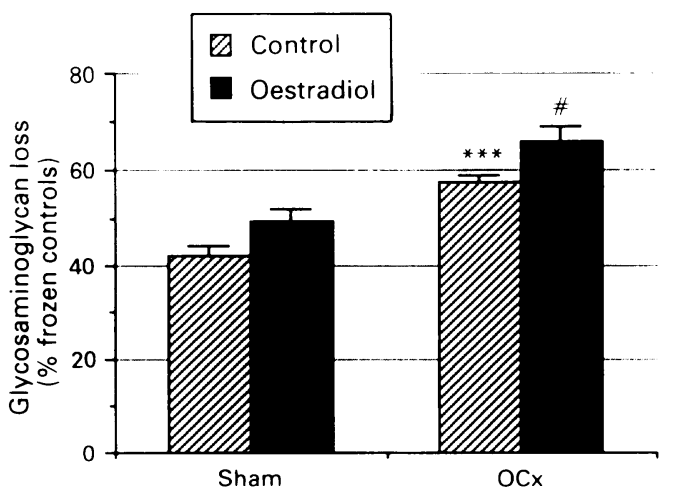

Figure 5 Effects of oestradiol treatment on inflammation induced glycosaminoglycan loss. Experimental design as in fig 4. Animals received biweekly subcutaneous injections of $0 \cdot 05 \mu \mathrm{g}$ oestradiol in oil (oestradiol) or oil alone (controls). There were eight to 10 animals in each group. ${ }^{\star \star \star} p<0 \cdot 001$ v control sham; \#p<0.05 v control $O C x$.

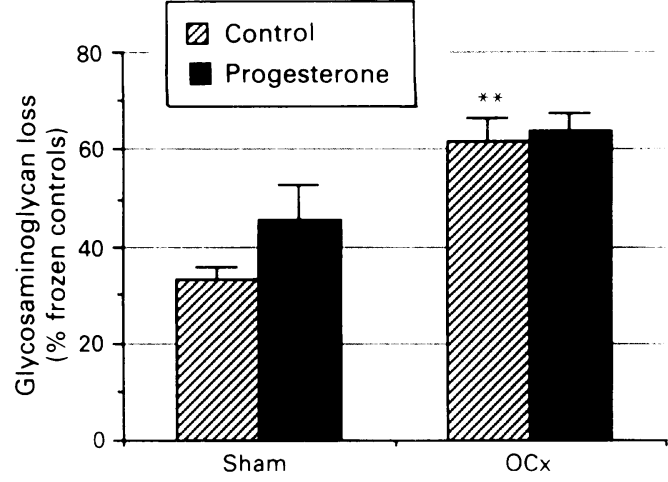

Figure 6 Effects of progesterone treatment on inflammation induced glycosaminoglycan loss.

Experimental design as in fig 4. Animals received a

subcutaneous silastic capsule either empty (controls) or filled with crystalline progesterone. There were eight to 10 animals in each group. ${ }^{\star *} p<0.01 \mathrm{v}$ control sham.

animals which had undergone orchidectomy with oestradiol and progesterone. Oestradiol significantly increased GAG loss in animals which had had an orchidectomy $(65.8(3.5) v$ $57 \cdot 7(1.6) \%$ loss; $p<0 \cdot 05)$. Treatment with progesterone did not induce significant changes in the rate of cartilage degradation, either in sham operated or castrated male animals, though there was a tendency to accelerate GAG loss in the former group. Combined treatment with oestradiol and progesterone did not result in significant changes compared with control animals (data not shown).

EFFECTS OF ORCHIDECTOMY ON GRANUIOMA FORMATION

To assess the effects of orchidectomy on the development of granulomatous tissue, the fluid content and dry tissue mass of three week granulomas from animals which had had an orchidectomy and sham operated controls from five different experiments were examined together (table 1). No significant effects of castration were seen in either parameter. Results were similar in each individual experiment and treatment with the various sex hormones did not change granuloma formation (data not shown).

EX VIVO PRODUCIION OF INTERLEUKIN $1 \mathrm{BY}$ GRANULOMATOUS TISSUF

Granulomatous tissue dissected 10 days after implantation and cultured for 48 hours released measurable amounts of IL $-1 \alpha$. The total amount released into the supernatant was

Table 1 Effects of orchidectomy on fluid volumes and dry tissue mass of cotton induced granulomas, three weeks after implantation. Results (mean (SEM)) from 40-42 individual animals in control sham and orchidectomised groups from five different experiments were analysed together. None of the differences reached statistical significance (Student's $t$ test)

\begin{tabular}{lll}
\hline & $\begin{array}{l}\text { Fluid } \\
(m g)\end{array}$ & $\begin{array}{l}\text { Dry mass } \\
(m g)\end{array}$ \\
\hline Sham operation & $38.73(1 \cdot 0)$ & $6.94(0.21)$ \\
Orchidectomy & $40.2(1 \cdot 06)$ & $7 \cdot 12(0.22)$ \\
\hline
\end{tabular}


Table 2 Effects of sex hormones on cartilage degradation and proteoglycan synthesis of cultured cartilage. Femoral head cartilages from male Wistar rats were cultured for 10 days in Dulbecco's modified Eagle's medium (DMEM) in the presence of various concentrations of testosterone, oestradiol, progesterone, or vehicle alone (DMSO). The percentage degradation was calculated as described under Methods. Results presented as mean (SEM)

\begin{tabular}{|c|c|c|c|}
\hline & $\begin{array}{l}\text { Hormone } \\
\text { concentration } \\
\text { (moll) }\end{array}$ & $\begin{array}{l}\text { Degradation } \\
(\%)\end{array}$ & $\begin{array}{l}\text { Incorporation } \\
\text { of sulphur-35 } \\
\left(\mathrm{cpm} \times 10^{-3} / \mathrm{mg}\right)\end{array}$ \\
\hline DMEM/DMSO $(n=8)$ & - & $27 \cdot 1(1 \cdot 05)$ & $6.52(0.33)$ \\
\hline Medium + testosterone $(n=8)$ & $\begin{array}{l}1 \times 10^{-9} \\
1 \times 10^{-8 \star} \\
1 \times 10^{-7}\end{array}$ & $\begin{array}{l}23.9(0.84) \dagger \\
23.1(0.74) \ddagger \\
23.7(1.1) \dagger\end{array}$ & $\begin{array}{l}6.30(0.22) \\
6.59(0.30) \\
6.22(0.33)\end{array}$ \\
\hline Medium+oestradiol $(n=8)$ & $\begin{array}{l}2.5 \times 10^{-12} \\
2.5 \times 10^{-11 \star} \\
2.5 \times 10^{-10}\end{array}$ & $\begin{array}{l}23.7(1.1) \dagger \\
24.7(0.75) \\
22 \cdot 1(0.92) \ddagger \\
22.9(1.3) \dagger\end{array}$ & $\begin{array}{l}6.22(0.33) \\
6.54(0.31) \\
6.48(0.29) \\
5.97(0.21)\end{array}$ \\
\hline Medium+ progesterone $(n=8)$ & $\begin{array}{l}1 \times 10^{-9} \\
1 \times 10^{-8 \star} \\
1 \times 10^{-7}\end{array}$ & $\begin{array}{l}20.7(0.74) \oint \\
20.9(1.36) \dagger \\
23.9(1.04)\end{array}$ & $\begin{array}{l}6 \cdot 16(0 \cdot 16) \\
6 \cdot 74(0 \cdot 16) \\
6 \cdot 70(0 \cdot 19)\end{array}$ \\
\hline
\end{tabular}

*Average physiological levels for male animals

$\mathrm{tp}<0.05 ; \neq \mathrm{p}<0 \cdot 01 ; \mathrm{g} \mathrm{p}<0 \cdot 001$, all $v$ DMEM/DMSO.

significantly higher for granulomas from male animals which had had an orchidectomy (13.8 $(1 \cdot 21) \mathrm{pg} / \mathrm{mg}$ dry mass; nine animals) compared with those from eight sham operated animals $(8.3(0.65) ; \mathrm{p}=0.002)$.

IN VITRO EFFECTS OF SEX HORMONES ON CARTILAGE PROTEOGLYCAN SYNTHESIS AND DEGRADATION

Table 2 shows that all three hormones (testosterone, oestradiol, and progesterone) induced a significant reduction of the rate of spontaneous GAG release into the supernatant when tested in physiological concentrations for male rats. Effects were more marked with progesterone, which, at a concentration of 1 $\mathrm{mmol} / 1$, reduced GAG release from $27 \cdot 1 \%$ in controls to $20.7 \%(p<0.001)$. These effects were not dose dependent. None of the sex hormones influenced significantly the rate of proteoglycan synthesis as measured by incorporation of sulphur- 35 .

IN VITRO INTERACTION OF SEX HORMONES WITH INTERLEUKIN $1 \alpha$ ON CARTILAGE PROTEOGLYCAN SYNTHESIS AND DEGRADATION The addition of $1 \mathrm{ng} / \mathrm{ml} \mathrm{mrIL-1} \alpha$ during the second five day culture period resulted in a significant increase of cartilage degradation $(37.0 v 27 \cdot 1 \% ; \mathrm{p}<0.01)$ and a decrease in

Table 3 Interaction of sex hormones with interleukin (IL-1) effects on cultured cartilage. Femoral head cartilages from male Wistar rats were cultured in the presence of sex steroids or dimethylsulphoxide (DMSO) alone for 10 days with one change of medium at day 5. Mouse recombinant $I L-1 \alpha(1 \mathrm{ng} / \mathrm{ml})$ was added to the medium for the second culture period. None of the results for the hormone treated groups was significantly different from IL-1/DMSO

\begin{tabular}{|c|c|c|c|}
\hline & $\begin{array}{l}\text { Hormone } \\
\text { concentration } \\
\text { (molll) }\end{array}$ & $\begin{array}{l}\text { Degradation } \\
(\%)\end{array}$ & $\begin{array}{l}\text { Incorporation } \\
\text { of sulphur-35 } \\
\left(\mathrm{cpm} \times 10^{-3} / \mathrm{mg}\right)\end{array}$ \\
\hline DMEM $\star / D M S O$ & & $27 \cdot 1(1 \cdot 05)$ & $6.52(0.33)$ \\
\hline IL-1 $\alpha /$ & - & $37 \cdot 0(2 \cdot 3) \dagger$ & $3.76(0.34) \ddagger$ \\
\hline \multirow[t]{3}{*}{$\mathrm{IL}-1+$ testosterone $(\mathrm{n}=8)$} & $1 \times 10^{-9}$ & $37.5(1.37)$ & $4.34(0 \cdot 30)$ \\
\hline & $1 \times 10^{-8}$ & $37.5(1.5)$ & $3.87(0.26)$ \\
\hline & $1 \times 10^{-7}$ & $37 \cdot 8(1 \cdot 37)$ & $4.78(0.38)$ \\
\hline \multirow[t]{3}{*}{ IL-1 +oestradiol $(n=8)$} & $2.5 \times 10^{-12}$ & $35.6(1.3)$ & $4.72(0.28)$ \\
\hline & $2.5 \times 10^{-11}$ & $36.7(0.97)$ & $4.51(0.29)$ \\
\hline & $2.5 \times 10^{-10}$ & $41 \cdot 0(1 \cdot 32)$ & $4.05(0.39)$ \\
\hline \multirow[t]{3}{*}{ IL-1+progesterone $(n=8)$} & $1 \times 10^{-9}$ & $36.5(2 \cdot 19)$ & $5.05(0.50)$ \\
\hline & $1 \times 10^{-8}$ & $35.4(1.73)$ & $4.46(0.48)$ \\
\hline & $1 \times 10^{-7}$ & $39.7(1.02)$ & $3.94(0.47)$ \\
\hline
\end{tabular}

ॠMEM=Dulbecco's modified Eagle's medium.

tp<0.01 $v$ DMEM/DMSO

$\neq \mathrm{p}<0.001 v$ DMEM/DMSO. proteoglycan synthesis $\left(3.76 v 6.52 \times 10^{3} \mathrm{cpm} /\right.$ mg; $\mathrm{p}<0.001)$. The effects of IL-1 on the two parameters were not significantly changed by preincubation and the continuous presence of sex hormones (table 3 ).

\section{Discussion}

Wrapping cartilage in cotton induces the formation of granulomatous tissue and a marked increase in cartilage damage, providing a suitable model to assess the effects of inflammation on cartilage. We have no clear explanation of the increase in GAG content observed at the end of the first week. It is possible, however, that the trauma of the operation and exposure to growth factors in serum play a part by accelerating the rate of proteoglycan synthesis above the negative effects of early inflammation. Differences between wrapped and non-wrapped cartilages at one week were not statistically significant and although they may have contributed to the differences seen at later points, these are much greater and increase with the time of implantation. Orchidectomy consistently resulted in an accelerated rate of cartilage loss in the presence of granulomatous reaction, coinciding with an increased production of IL-1 The effects of orchidectomy were completely nullified by the replacement of physiological levels of dihydrotestosterone, thus confirming the protective role of androgens in this process. The lack of further protection when dihydrotestosterone was given to sham operated animals shows that the maximum androgen mediated effects in this model were achieved at physiological levels.

Progesterone did not have any significant effect but oestradiol caused a significant increase in cartilage loss in animals which had had an orchidectomy but not in sham operated male animals, suggesting that androgens may counteract the deleterious effects of oestradiol. The levels of progesterone, and presumably oestradiol, achieved by our treatment regimens were supraphysiological for male mice, but our results nevertheless show that these hormones do not offer protection against cartilage degradation in the male animal, despite their known immunosuppressive actions. These results corroborate observations of increased susceptibility and severity of streptococcal cell wall arthritis after castration or oestrogen treatment in male LEW/N rats. ${ }^{10}$

Investigations of the effects of sex hormones in inflammatory disorders face a number of methodological difficulties derived mostly from the complexity of hormone regulation and metabolism and the intricate interactions between the gonadal, adrenal, and immune systems. ${ }^{411}$ In vivo models allow the assessment of end results that cannot be directly predicted from simplified in vitro systems. The model we used is not intended as a direct reproduction of RA but as a model of cartilage damage induced by granulomatous tissue with similarities to rheumatoid pannus. Its features have been well described and published results on the effects of current treatments for 
rheumatic diseases have shown a good correlation with experience in human disease and other experimental models. ${ }^{12}$ Unlike more conventional models it allows a reproducible measurement of cartilage destruction offering appropriate conditions for the study of this essential component of arthritis.

The peripheral transformation of testosterone into oestradiol has been shown to be an important confounding factor on studies addressing immunological effects of sex hormones. ${ }^{4}$ Our results show that prolonged testosterone treatment of experimental male animals, in a dose sufficient to attain physiological levels, will result in extremely high oestradiol levels due to peripheral transformation and is, therefore, inadequate to study the effects of androgens. The silastic capsules of $5 \alpha$-dihydrotestosterone used here allowed stable androgen levels to be obtained in the low physiological range (compensating for the higher androgenic activity of dihydrotestosterone) without any detectable change in oestradiol levels.

Unsuspected sex hormone action is also a potential problem in in vitro studies. We have repeatedly found high levels of oestradiol and progesterone in different batches of fetal calf serum. Progesterone is also a common component of most available serum alternatives for culture purposes. Weak oestrogenic properties of phenol red, a common $\mathrm{pH}$ indicator in culture media, have been shown repeatedly and we have therefore avoided these components in our culture conditions.

The mechanisms of the protective actions of androgens are still unclear. The lack of influence of orchidectomy on non-wrapped cartilage suggests that the effects of sex hormones are mainly due to actions on the inflammatory tissue and not directly on the chondrocytes. To clarify this point further we investigated the effects of sex steroids and their interaction with IL-1 on cartilage metabolism in vitro. The effects seen on spontaneous proteoglycan loss in culture are common to the different sex steroids studied and do not seem to be important in vivo. None of the sex hormones showed any significant interference with IL-1 induced effects on proteoglycan synthesis and degradation in vitro, reinforcing the concept that direct actions on cartilage do not contribute significantly to the overall effects seen in the in vivo model. The lack of dose dependency of sex hormone effects on cartilage is probably related to the number and kinetics of the respective receptors in this tissue. To our knowledge only oestradiol receptors have been described in articular chondrocytes. ${ }^{13} 14$ These receptors were found in small amounts but showed high affinity, which may justify saturation at physiological concentrations. It should also be noted that the lower concentrations used in our in vitro studies correspond approximately to physiological concentrations of free sex steroids, given that these hormones are bound to carrier proteins in excess of $90 \%$.

Orchidectomy did not affect parameters of granulomatous tissue development, in agree- ment with previous findings with this model showing a lack of positive association between the mechanisms responsible for generating acute exudative inflammation and those causing cartilage destruction. ${ }^{7}$ A similar dissociation has been proposed for rheumatoid arthritis. ${ }^{15}$ Our results show that sex steroids affect the erosive ability of granulomatous tissue, probably by influencing the production of mediators, as suggested by the increased release of IL-1 associated with orchidectomy. Although we cannot provide firm evidence that IL-1 is responsible for the acceleration of cartilage loss induced by castration, there is overwhelming evidence for a pivotal role of this cytokine in cartilage degradation in human and experimental disease and our own results in vitro show that mouse IL- $1 \alpha$ is capable of inducing increased loss and decreased synthesis of proteoglycan from rat FHCs. It is not clear how androgens should bring about this effect. In our experience, orchidectomy does not significantly influence the ex vivo release of IL- 1 by resident mouse peritoneal macrophages (data not shown). Macrophages in an inflammatory environment, however, may show a different response and the complex interactions that surround these cells in inflammatory tissue provide a large number of pathways for indirect modulation-for example, through $\mathrm{T}$ cells and lymphokine production. ${ }^{16}$ The possibility of direct androgen actions on these cells is supported by the description of androgen receptors in macrophage-like cells in normal and rheumatoid synovia. ${ }^{5}$ It is unlikely that the effects of androgens in this model are dependent on any interaction with the hypothalamus-pituitaryadrenal axis, as treatment with dihydrotestosterone results in reduced glucocorticoid response to the inflammatory process. ${ }^{17}$ This suggests a direct anti-inflammatory role for androgens, which is also supported by previous findings of reduced inflammation and cartilage erosion after intralesional administration of testosterone in models of air pouch and antigen induced arthritis in mice. ${ }^{18}$

In summary, our results show that androgens have a protective role against inflammation induced cartilage degradation in male animals, probably mediated by interference with the immune and inflammatory process. These results offer additional support for a potential pathogenic role of androgens in human disease and suggest that in addition to ameliorating disease activity, androgens may contribute to reduce joint destruction and disability in arthritis.

Dr Da Silva is a visiting physician from Coimbra, Portugal, under a grant from INVOTAM (Lisbon). Dr Larbre is supported by a grant from INSERM, France.

1 Gordon C, Beastall G H, Thomson J A, Sturrock R D. Androgenic status and sexual function in males with Androgenic status and sexual function in males with
rheumatoid arthritis and ankylosing spondylitis. $Q \mathcal{M} \mathrm{Med}$ 1986; 60: 671-9.

2 Spector T D, Ollier W, Perry L A, et al. Free and serum testosterone levels in 276 males: a comparative study of
tector T D , Ollier W, Perry L A, et al Free and serum testosterone levels in 276 males: a comparative study of
rheumatoid arthritis, ankylosing spondylitis and healthy rheumatoid arthritis, ankylosing spondylitis

3 Cutolo M, Balleari E, Giusti M, Intra E, Accardo S. Androgen replacement therapy in male patients with rheumatoid arthritis. Arthritis Rheum 1991; 34: 1-5. 
4 Ansar Ahmed S, Penhale W J, Talal N. Sex hormones, immune responses, and autoimmune diseases. Mechanisms of sex hormone action. Am f Pathol 1985; 121: 531-51.

5 Cutolo M, Accardo S. Villaggio B, et al. Evidence for the presence of androgen receptors in the synovial tissue of presence of androgen receptors in the synovial tissue of
rheumatoid arthritis patients and healthy controls. rheumatoid arthritis patients and

6 Franchimont P, Bassleer C. Effects of hormones and local growth factors on articular chondrocyte metabolism. f Rheurat 1991; 18 (suppl 27): 68-70.

7 De Brito F B, Moore A R, Holmes M J G, Willoughby D A. Cartilage damage by a granulomatous reaction in a murine species. Br f Exp Pathol 1987; 68: 675-86.

8 Wathen N C, Perry L A, Rubenstein E, Chart T. A relationship between sex hormone binding globulin and dehydroepiandrosterone sulphate in normally menstruating females. Gynaecol Endocrinol 1987; 1: 47-50.

9 Farndale R W, Buttle D J, Barrett A J. Improved quantitation and discrimination of sulphated glycosaminoglycans by use of dimethylmethylene blue. Biochim Biophys Acta 1986; 883: 173-7.

10 Allen J B, Blatter D, Calandra G B, Wilder R L. Sex hormonal effects on the severity of streptococcal cell wallinduced polyarthritis in the rat. Arthritis Rheum 1983; 26: induced 360 .

11 Lesniewska B, Miskowiak B, Nowak M, Malendowicz L K. Sex differences in adrenocortical structure and function.
XXVII. The effect of ether stress on ACTH and corticosterone in intact, gonadectomized, and testosteroneor estradiol-replaced rats. Res Exp Med 1990; 190: 95-103.

12 Bottomley K M K, Griffiths R J, Rising T J, Steward A. A modified air pouch model for evaluating the effects of modified air pouch model for evaluating the effects of compounds on granuloma induced cartilage degradation.

Br F Pharmacol 1988; 93: 627-35.
13 Rosner I A, Manni A, Malemud C J, Boja B, Moskowitz $\mathbf{R}$ W. Estradiol receptors in articular chondrocytes. Biochem Biophys Res Commun 1982; 106: 1378-82.

14 Young P C M, Stack M T. Estrogen and glucocorticoid receptors in adult canine articular cartilage. Arthritis Rheum 1982; 25: 568-73.

15 Krane S M, Simon L S. Rheumatoid arthritis: clinical features and pathogenetic mechanisms. Med Clin North Am 1986; 70: 263-77.

16 Araneo B A, Dowell T, Diegel M, Daynes R A. Dihydrotestosterone exerts a depressive influence on the production of interleukin-4 (IL-4), IL-5 and $\gamma$-interferon, but not IL-2 by activated murine T cells. Blood 1991; 78: 688-99.

17 Da Silva J A P, Peers $S H$, Perretti $M$, Willoughby D A Sex steroids affect glucocorticoid response to chronic Sex steroids affect glucocorticoid response to chronic
inflammation and to interleukin $1 . \mathcal{F}$ Endocrinol. In press.

18 Steward A, Bayley D L. Effects of androgens in models of rheumatoid arthritis. Agents Actions 1992; 35: 268-72. 